\title{
El precio de la palabra: la hegemonía del capitalismo electrónico-informático \\ y el googleísmo
}

\author{
GUSTAVO LINS RIBEIRO
}

Me interesa comprender cómo los empresarios e innovadores de internet se han convertido en poderosos capitalistas. La mercantilización de las palabras, el mercado virtual global, la economía de la carnada y las economías del enlace, del clic y del me gusta son modos de producir ganancias, cruciales para entender el rápido crecimiento de algunas de las principales empresas del presente. También argumento que el googleísmo es para el capitalismo contemporáneo lo que fueron el fordismo y el toyotismo: modelos de gerenciamiento de los factores de producción que se diseminan en todo el globo. La innovación masiva en línea o crowdsourcing se presenta como un modo de apropiarse de la creatividad en línea, básico para la (re)producción del capitalismo electrónico-informático.

PALABRAS CLAVE: Google, capitalismo, crowdsourcing, innovación, mercantilización

The Price of the Word. The Hegemony of Computer-Electronic Capitalism and Googleism

I am interested in understanding how internet entrepreneurs and innovators have become powerful capitalists. The commodification of words, the virtual global market, the "bait economy" and the link/like/hit economies are modes of profit making that are crucial to understand the rapid growth of some of the leading corporations of our times. I also argue that Googleism is to current capitalism what Fordism and Toyotism were: models of managing factors of production that are globally disseminated. Crowdsourcing, or massive innovation on line, is seen as a mode of appropriating online creativity basic to the reproduction of electronic-computer capitalism. 
n 1998 escribí sobre internet como la "base tecnosimbólica” que por medio de la "política cibercultural", el "testimonio a distancia" y el "activismo político a distancia" podría generar "comunidades transnacionales imaginadasvirtuales” (Lins Ribeiro, 1998). Más tarde (Lins Ribeiro, 2003), escribí sobre las relaciones entre el "espacio público virtual" y el "espacio público real". En este artículo, dada la centralidad de internet para el desarrollo de algunas de las más grandes compañías del mundo, como Google y Facebook, me interesa entender cómo es que empresarios e innovadores de internet se transforman en poderosos capitalistas. Cómo el capital financiero ha usado internet para incrementar su capacidad económica; cómo han crecido las industrias de software y de aparatos digitales y electrónicos, o cómo ha transformado internet muchas actividades económicas, son temas muy importantes que, no obstante, no constituyen aquí mi principal preocupación.

Mis intereses se relacionan con una literatura bastante amplia, que crece cada día. Algunos autores, con intereses parecidos o distintos, hablan de una "economía informacional" (Castells, 1996), de un "capitalismo digital" (Schiller, 2000) y de un "capitalismo cognitivo" (Cocco, Silva y Patez Galvão, 2003; Fumagalli, 2010; Moulier-Boutang, 2008; 2010; Blondeau et al., 2004; Vercellone, 2011). De hecho, la historia de este campo de conocimiento se remontaría hasta las décadas de 1960 y 1970, cuando tuvieron lugar los debates sobre la sociedad posindustrial y la sociedad de la información (Albagli y Maciel, 2010: 4). La crisis del fordismo y el taylorismo en la década de 1970 abrió un espacio para "la flexibilización de los procesos productivos y del trabajo mismo" (2010: 4), lo que generó un ambiente favorable para la expansión de las tecnologías de información y comunicación, así como de otras tecnologías "altamente intensivas en información y conocimiento, con implicaciones directas en el proceso productivo" (2010: 4). En la década de 1980, algunos economistas hablaron de la existencia de una economía del conocimiento y el aprendizaje (2010: 4).

El debate sobre el capitalismo cognitivo es quizá el más amplio de todos. Se centra en "la profunda mutación que afecta la forma en que el capital es dotado de valor" (Corsani, 2003: 15, citado en Albagli y Maciel, 2010: 7), y pone énfasis en "la nueva centralidad del trabajo inmaterial — que moviliza información, 
conocimiento, imágenes, creatividad, afectos y relaciones_-" (Albagli y Maciel, 2010: 7). Aquí también se ve la innovación como "algo que no está restringido al ambiente de la empresa, sino que, por el contrario, involucra al tejido social como un todo" (2010: 9). En este nuevo sistema, "la extracción de ganancias basada en el conocimiento y en la innovación se convierte en el factor central de la acumulación” (Moulier-Boutang, 2010: 64). La "hipótesis del capitalismo cognitivo" brinda un marco interpretativo interesante para pensar en las dinámicas económicas actuales. Sin embargo, exagera la novedad de la expropiación del conocimiento por parte de los capitalistas, algo que claramente sucedió hace algunos siglos, cuando ocurrió la transición de los sistemas de manufactura a los sistemas industriales. La apropiación de la creatividad de los artesanos y manufactureros por parte de los capitalistas es un proceso anterior a la Revolución industrial y alcanzó su ápice en el siglo XIX, con la institucionalización de la invención como un medio para desarrollar innovaciones de manera profesional en los laboratorios, para adquirir patentes que a su vez garantizarían nichos monopólicos a las empresas y los capitalistas-inventores (Noble, 1977; Thomson, 2009). En este sentido, el capitalismo industrial ha sido siempre cognitivo y la centralidad de la innovación como método para incrementar la acumulación y la diferenciación capitalistas puede rastrearse hasta, por lo menos, el siglo XIX. Además, como antropólogo, no puedo más que estar de acuerdo con la famosa afirmación de Antonio Gramsci sobre la imposibilidad de separar al Homo faber del Homo sapiens y con la crítica que hace Dan Schiller de las nociones de "sociedad de la información" y "trabajo inmaterial":

Si, al contrario de la visión recibida de los teóricos de la sociedad de la información, todos los trabajadores tienen capacidad mental, entonces distinguir formas particulares de trabajo como particularmente "inmateriales" o "comunicativas" debe ser un error [...] todo trabajo contiene un aspecto de procesamiento intelectual o de información [...] (2016: 6).

El error categórico no radica en la reducción de la manufactura o de cualquier tipo de trabajo, de hecho, a un grado más bajo, sino en el inequívoco ascenso de una (supuesta) especie de trabajo concreto a un desiderátum [...]. Construir una teoría de la sociedad basada en cualquier forma de trabajo concreto invalida y resta importancia a las contribuciones sustantivas de todos los otros tipos de trabajo concreto. Es arbitrario y teóricamente injustificado (2016: 8).

Por ello me refiero a la hegemonía del capitalismo electrónico-informático, para dejar claro que, aunque estemos experimentando una nueva hegemonía industrial, no puede subestimarse la importancia de otros sectores y actividades económicas. Pero es cierto que la difusión de las computadoras y de internet ha cambiado los modos de apropiación del conocimiento, en especial del conocimiento colectivo. También es cierto que la apropiación del conocimiento y la creatividad colectivos no es la única manera de producir valor dentro del capitalismo electrónico-informático. Como veremos, la mercantilización de las palabras, el mercado global virtual, la "economía de la carnada" y la "economía del me gusta y del clic" son formas de producir ganancias que resultan cruciales para comprender el rápido crecimiento de algunas de las principales compañías de nuestros tiempos.

Uso la expresión capitalismo electrónicoinformático inspirado en Benedict Anderson (1983), quien, en su célebre libro Comunidades imaginadas, escribió sobre el capitalismo de imprenta. Acuñé esta expresión para subrayar la hegemonía de un sector industrial y económico representada por la centralidad de internet y sus sistemas relacionados en la economía política capitalista actual. Elegí estas palabras 
para abrir el horizonte a discusiones más amplias sobre innovaciones tecnológicas, formas capitalistas de producción, organización, extracción de valor y acumulación. Ante todo, quiero llamar la atención sobre la máquina, la computadora, que es central para la "reorganización de las relaciones sociales" y la constitución de "nuevos dominios sociales", como afirman Robert Latham y Saskia Sassen (2005: 1). Podría decirse que Anderson y yo hablamos de subsistemas capitalistas con impactos profundos en todos los ámbitos de la vida económica, social, política y cultural.

\section{La hegemonía del capitalismo electrónico-informático}

Algunas innovaciones tecnológicas se ven como hitos que cambian el curso de la historia humana. Así, pasan a ser mucho más que máquinas, aparatos o sistemas: se convierten en formas de estar en el mundo. Estas innovaciones sirven de base a nuevas actividades lucrativas, nuevos modelos gerenciales y productivos, nuevos discursos y también a nuevas visiones trascendentales de la sociedad y la producción de hegemonía. El capitalismo electrónico-informático y sus tecnologías son un ejemplo emblemático. Las fuerzas que subyacen a esta hegemonía son, a saber, las tasas de retorno excepcionales generadas por las innovaciones de vanguardia y por las prácticas gerenciales y productivas; su capacidad para obtener cantidades extraordinarias de valor de recursos que antes estaban fuera del alcance de los capitalistas, y los nichos monopólicos que garantizan por medio de marcas registradas y copyrights.

Nos dicen que estamos en la era digital, en la sociedad de la información o del conocimiento. De hecho, con la hegemonía del capitalismo electrónico-informático es posible la rápida diseminación y reproducción de un nuevo zeitgeist, de sus necesidades, su centralidad en la producción y el consumo, concomitante a la elaboración de nuevas ideologías y utopías, y reinterpretaciones del pasado y el futuro. Todos estos cambios se expresan también en cambios de la infraestructura material - la proliferación de cables y antenas, por ejemplo- y en el gran aumento de la disponibilidad de aparatos que los consumidores necesitan para formar parte del nuevo mundo. En la actualidad, la convergencia tecnológica hizo crecer mucho el número de smartphones. En 2015, había 6.1 mil millones de teléfonos inteligentes. Se espera que para 2020 la cantidad de celulares ascienda a 9.2 mil millones (Lunden, 2015). Dentro de no mucho tiempo, cada persona será un smartphone.

El uso del término "hegemonía" tiene una doble implicación. Por un lado, significa la aceptación silenciosa, generalizada y naturalizada del predominio del capitalismo electrónico-informático. Por otro lado, implica la existencia de otras fuerzas, viejas y nuevas, que también están presentes, casi siempre de manera subordinada, en el interior del mismo campo de poder (Bourdieu, 1986), dominado por el capitalismo electrónico-informático. ${ }^{1}$ De hecho, en cualquier coyuntura económica, los sectores económicos de punta coexisten con muchos otros.

Desde la Revolución industrial, la historia industrial se ha amalgamado con la historia de la ciencia y la tecnología, sobre todo en lo que concierne a

En la política, la noción gramsciana de hegemonía señala la capacidad de unificar posiciones por medio de la fuerza y el consenso (Gramsci, 2006; Macciocchi, 1974). Para Williams, la noción de hegemonía procura "describir un predominio más generalizado que incluye, como una de sus características principales, una manera particular de ver el mundo y las relaciones hombre/naturaleza" (1983: 144). En mi análisis subrayo la hegemonía como dirección, dominio y liderazgo construidos por medio de la persuasión y el consenso. Bourdieu (1986) define un campo como una serie de relaciones e interrelaciones basadas en valores y prácticas específicos que operan en contextos determinados. Un campo es heterogéneo por definición, está conformado por distintos actores, instituciones, discursos y fuerzas en tensión. 


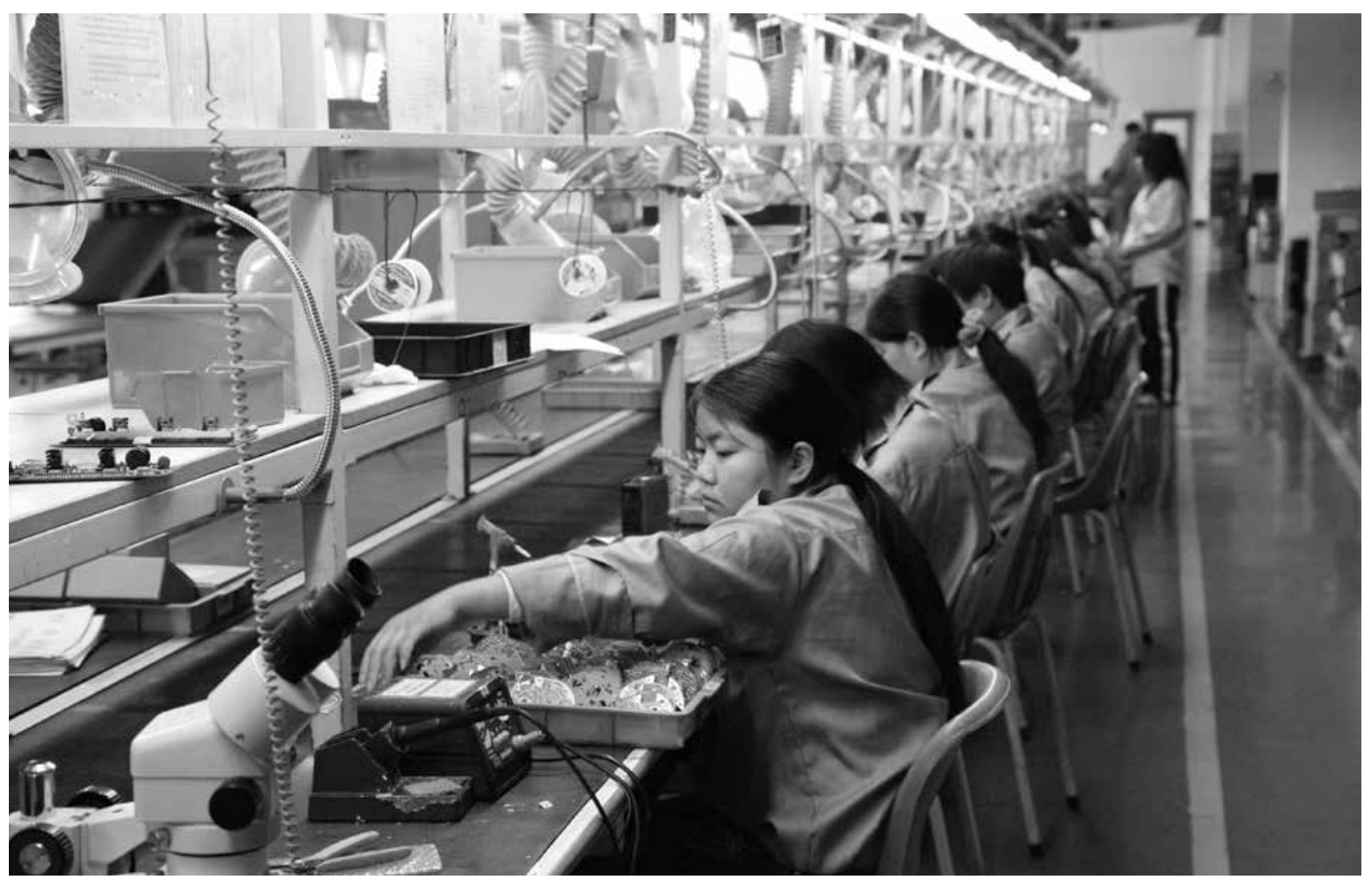

Walter H. GonzÁlez P Planta de Echo Electronics HK, Hong Kong, China, diciembre de 2008.

los modos de incrementar la productividad del trabajo, el dominio sobre las fuerzas naturales, el uso de la energía y la logística de todos los factores de producción. Durante los últimos 200 años, las máquinas y la mecanización, los sistemas centrales de la era mecánica, se han vuelto tan omnipresentes que, ya en 1934, Lewis Mumford decía que “dominaban todos los aspectos de nuestra existencia” (1950: 4). Mumford se preguntaba: “¿cómo es que la máquina ha podido adueñarse de la sociedad europea hasta que, por una adaptación interna, esa misma sociedad se rindió ante la máquina?” (1950: 4). En la historia de la tecnología y la industria, es difícil definir cuándo empezó la "era mecánica”, esto es, cuándo empezaron los mecanismos a ser la fuente hegemónica de la producción y las metáforas. Pero su decadencia como grandes fuerzas de la vida económica, social, política y cultural puede relacionarse con la creciente importancia de la electricidad en el último tercio del siglo XIX. La hegemonía de la electricidad y la electrónica llevó a una nueva clasificación histórica, la "era electrónica", que se asocia a lo que Manuel Castells llama un "nuevo paradigma tecnológico":

El núcleo científico y técnico de este paradigma es la microelectrónica, y se apoya en los descubrimientos sucesivos del transistor (1947), el circuito integrado (1957), el proceso planar (1959) y el microprocesador (1971). Las computadoras [...] revolucionaron el procesamiento de datos, tanto a nivel del hardware como a nivel del software. Las telecomunicaciones se convirtieron en el vector clave para la difusión y la plena utilización de las nuevas tecnologías permitiendo las conexiones entre unidades de procesamiento y la formación de sistemas de información (1989: 12). 
No es posible comprender el papel de las fábricas de textiles en el desarrollo del capitalismo industrial sin hacer referencia a la ciudad de Manchester, Inglaterra (véase, por ejemplo, Engels, 2009). En el mismo sentido, no es posible entender la hegemonía del capitalismo electrónico-informático sin considerar su Meca, Silicon Valley, en Estados Unidos. Este valle del norte de California es el principal centro global del capitalismo electrónicoinformático. Hace más de 20 años, Manuel Castells escribió que allí se desarrollaron "el circuito integrado, el microprocesador y la microcomputadora, entre otras tecnologías clave"; allí "late, desde hace cuatro décadas, el corazón de la innovación electrónica" (1996: 53):

[Silicon Valley] se formó como un medio de innovación por la convergencia, en un mismo sitio, de: nuevos conocimientos tecnológicos; una gran oferta de ingenieros y científicos de las universidades más importantes de la zona; el financiamiento generoso de un mercado asegurado mediante el Departamento de Defensa; y, en sus primeros momentos, el liderazgo institucional de la Universidad de Stanford [...]; la creación continua de firmas start-up apuntaló a Silicon Valley como el centro mundial de la microelectrónica a principios de la década de 1970 (1996: 54, 55).

El valle pronto se convirtió en el cluster de alta tecnología más grande e importante del mundo, un modelo a ser imitado por todos. También es el paraíso de los empleos flexibles, un mercado de trabajo segmentado de manera étnica, en el que las compañías contratan un número mínimo de personal permanente, en el que abundan los intermediarios, y en consecuencia, los salarios y carreras para la mayoría son escasos. La explotación, el desempleo, la desigualdad y la inseguridad también son comunes en Silicon Valley (Benner, 2002). Para Saxenian, se trata de un escenario de creciente desigualdad y la hegemonía mundial del valle está menos amenazada por la "competencia extranjera" que por las "décadas de falta de atención en lo que respecta al desarrollo humano, social y colectivo que sustenta su éxito económico" (2016: 47).

De todos los modos, al asociarse con figuras míticas como Bill Gates - Microsoft-, Steve Jobs y Steve Wozniak —Apple-, Silicon Valley sigue atrayendo jóvenes talentos de todo el mundo. Muchos sueñan con ser Larry Page o Sergei Brin, los fundadores de Google, o Mark Zuckerberg, el fundador de Facebook, los grandes modelos de inspiración más recientes en el panteón del capitalismo electrónico-informático.

La mística del espíritu empresarial estadounidense está llena de venerados genios legendarios, magnates "visionarios" que, con sus poderosos insights y su inventiva, han dado forma al destino de la humanidad y se han convertido en los fundadores de extraordinarios imperios industriales o financieros. Las últimas décadas del siglo XIX constituyen el momento arqueológico del capitalismo electrónicoinformático: un periodo en el que la electricidad se establecía como una fuente masiva y viable de energía para los consumidores individuales e industriales. El nombre más célebre de aquellos días fue el de Thomas Edison, el hombre que popularizó los sistemas de iluminación incandescente y "deliberadamente" hizo que la "invención dejara de ser un fenómeno aleatorio para convertirse en un oficio rutinario y profesional [...] inextricablemente determinado por consideraciones económicas” (Noble, 1977: 8). Edison creó un "laboratorio bien equipado", así como compañías poderosas. Él fue uno de los fundadores de General Electric, la enorme corporación que contó también con la participación de J. P. Morgan, el financiero. Las "primera y segunda generaciones de los hombres que crearon y administraron la moderna industria eléctrica", entre 1880 y 1920, fueron la "vanguardia del desarrollo industrial basado en la ciencia en los 
Estados Unidos" (Noble, 1977: 7). La ciencia misma se transformó en capital, para usar las palabras de Harry Braverman (1974: 166) citadas en el libro clásico de David Noble (1977: 6), America by Design. Science, Technology and the Rise of Corporate Capitalism. Gracias a las leyes de patentes, las "invenciones" se convirtieron en la mejor forma de arrebatar nichos monopólicos en el mercado, el deseo de todo capitalista. La alianza entre inventores, ciencia, universidades, capital financiero, abogados de patentes y empresarios es parte de la arqueología del capitalismo electrónico-informático y se ha desarrollado mucho a partir de entonces. Los ingenieros siguen involucrados en las iniciativas de punta de Silicon Valley, pero a ellos se sumaron los matemáticos, sobre todo porque la métrica y los algoritmos juegan un papel central para las grandes corporaciones, como Google y Facebook.

\section{EL PRECIO DE LA PALABRA}

Desde que Google se fundó, en 1998, la historia de sus éxitos es en particular interesante por su papel central en el capitalismo electrónico-informático, la visión empresarial y gerencial que representa, y los ambiciosos objetivos que se impuso la compañía. Su nombre resulta familiar en todas partes. La autodefinición de la empresa revela cómo se ve a sí misma: "la misión de Google es organizar la información del mundo y hacerla universalmente accesible y útil". Google se ha convertido en un verbo, su deseo es ser ubicuo, omnipresente y omnisciente. Empezó como un motor de búsqueda y de manera gradual fue incorporando otra serie de servicios: Gmail (2004), Google Maps (2004), Google Earth (2005), Google Chrome (2008) y otros productos, muchos comprados por la compañía, como Waze y YouTube. Google Street View, una extensión de Google Maps, pretende ser una cristalización virtual del mapa de Jorge Luis Borges, un mapa del Imperio cuyo tamaño es el tamaño del Imperio. Pretende ser un panóptico virtual global de fácil acceso.

A pesar de los muchos servicios que ofrece Google, su motor de búsqueda sigue siendo la gran estrella en la constelación de la compañía. El acceso gratuito al servicio es una buena noticia para el usuario. ¡Perfecto! ¡Una de las compañías más grandes y más poderosas del mundo, con casi 40000 empleados en más de 70 países, ofrece un servicio esencial gratuito! Quizá Google sigue en sintonía con las fantasías hippies de muchos de los pioneros de internet, que comenzaron sus negocios en un pequeño garaje o en el dormitorio de la universidad, con la cabeza llena de ideas comunitarias. Pero googlee usted, por ejemplo, la palabra "hoteles". En 0.69 segundos aparecerán mil millones de resultados (consultado el 17 de marzo de 2016). Es seguro que usted no pasará toda su vida mirándolos todos. Entre los 12 primeros resultados se encuentran TripAdvisor - un sitio de viajes estadunidense- - y otros, como booking.com, hotels.com, Expedia y Travelocity. Olvídese de encontrar aquel hotelito acogedor cerca de la casa del amigo al que quiere visitar.

Entonces, ¿cómo gana dinero Google y cómo se hizo tan poderosa como motor de búsqueda? Cuando alguien googlea una palabra y el resultado de la búsqueda aparece en la pantalla, quien buscó no sabe que la clasificación de lo que aparece está económicamente estructurada. El orden jerárquico se subasta y los ofertantes compran prioridad y visibilidad. En la actualidad, puede existir una expresión como word mining - literalmente, minería de palabras - porque hay un mercado de palabras clave y porque el precio y el poder clasificatorio de las palabras varía según su frecuencia y el poder económico que subyace en ellas:

El término colocación preferente es empleado por las compañías para promover las páginas web en las respuestas a las consultas. Las organizaciones pagan a las compañías de motores de búsqueda para que 
sus sitios web aparezcan en las posiciones más altas en los resultados de las búsquedas, de tal manera que puedan recibir más accesos [hits, en el original]. Los accesos cuentan cuando se acumulan. En la economía de los accesos, las organizaciones esperan ganar dinero mediante la publicidad de banners y mediante la presencia demostrable en la red. El conteo de los accesos demuestra la presencia. Indica las medidas de popularidad y de confiabilidad del sitio (Rogers, 2002: 197, citado en Gerlitz y Helmond, 2011: 3).

Flores y hoteles son "palabras clave muy competitivas”, dice Diane Tang, una científica informática y matemática de Stanford a la que Google contrató para crear un mecanismo de...

publicidad de palabras-de-búsqueda análogo al mercado de acciones, llamado Índice de Precios de Palabras Clave. "Es como un índice de precios al consumidor", dice Tang, a quien internamente apodan la Reina de los Clics. "Pero en lugar de una canasta de artículos como pañales, cerveza y donas, tenemos palabras clave" (Levy, 2011: 118).

El hecho de que la publicidad de Google se ejerza en un mercado de subastas significa que su economía es más fluida: los vacíos se llenan [...]. Si este mes American Airlines y el Ritz no compran la palabra "París", otros anunciantes pueden hacerlo. El precio de esta palabra clave puede disminuir junto con la demanda, pero en la amplísima economía de Google, los precios de otras palabras clave [...] pueden subir (Jarvis, 2011: 69).

En realidad, las palabras se convirtieron en mercancías. Esto no significa que las palabras no tuvieran valor antes, lo tenían, pero de otra manera. La palabra escrita es el origen de las primeras regulaciones sobre el valor de la creación. El libro es la madre del copyright, que a su vez se encuentra en el origen de otras formas de propiedad intelectual. Así, desde hace bastante tiempo, las palabras - o mejor dicho, los conjuntos de palabras encadenadas bajo una forma literaria - tenían valor porque su composición revelaba una creación única, una capacidad de invención, una autoría. Hoy, los motores de búsqueda revelan que cualquier palabra que pueda asociarse a mercancías o servicios tiene un valor. En la actualidad, el precio de las palabras se encuentra desencarnado: ya no supone una creación literaria. Las palabras son realmente fetiches. Para venderlas, ya no es necesario el escritor-creador. Las palabras aisladas tienen un precio. El precio de la palabra, que antes existía bajo la forma de la creación literaria, se ha reducido al precio de la palabra convertida en un signo de bienes y servicios capitalistas negociables. El capitalismo electrónico-informático ha creado una nueva mercancía y los medios para explotar este recurso en varias lenguas. Al transformar las palabras en signos de búsqueda, Google sacó a la luz el mercado de la palabra y lo convirtió en un mercado mundial. Al mismo tiempo, concentró en su propia sede la capacidad de ver lo que ocurre en el mundo - $\mathrm{y}$ en la economía - y saber cuáles son los intereses de las personas y las compañías, para crear para sí misma un panóptico electrónico del mercado. También hizo que el mercado global fuera investigable de manera instantánea y potencialmente disponible para cada usuario. Al hacerlo, fomentó un mercado global virtual poblado por usuarios y no por consumidores. Si llamáramos consumidores a los usuarios, la relación desigual entre las compañías y sus clientes quedaría en evidencia, es decir, los consumidores obviamente están comprando algo, mientras que al usar Google reciben un servicio gratuito en apariencia.

\section{LA ECONOMÍA DE LA CARNADA: LOS USUARIOS COMO TRABAJADORES NO REMUNERADOS}

Además de ignorar que las palabras tienen precio y que entramos en una nueva era de la industria de la 
publicidad, los usuarios también ignoran que ellos mismos y la información que proporcionan son las mercancías que Google y otras poderosas compañías electrónico-informáticas venden. Los muchos miles de millones de personas que usan estos sistemas en todo el mundo son los mayores activos de las empresas, ya que éstas dependen de los anuncios para obtener ganancias y el valor que paga un anunciante "depende únicamente del número de personas que hacen clic" en su anuncio (Jarvis, 2011: 68). ${ }^{2}$ De hecho, cuando una persona hace clic en un anuncio, está trabajando para estas compañías. La apropiación gratuita de valor ha sido durante mucho tiempo un modo predilecto de acumulación de capital. En realidad, es la definición misma de plusvalía. Hoy ocurre muchos millones de veces por hora en internet, la red transnacional automatizada que cubre todo el planeta, sin que los productores de valor estén conscientes o se molesten por el hecho de ser una línea de producción virtual global y una cadena de valor que se está usando de forma gratuita y constante. ${ }^{3}$ Aún se discute si compañías como Google están explotando a los usuarios (Hesmondhalgh, 2016). En realidad, la transformación de los usuarios en productores de valor es coherente con la interpretación marxista de que el consumo también es producción (Schmidt, 1977: 104). Al final, Google se ha convertido en la agencia de publicidad más grande del mundo gracias al desarrollo de medios complejos, sobre todo basados en estadísticas y algoritmos, que permiten que usuarios, anunciantes y proveedores coincidan (Levy, 2011: 69-120).

La falsa idea de que alguien obtiene un servicio sofisticado y muy necesario de manera gratuita subyace en lo que llamo la "economía de la carnada", que significa que se nos ofrece un regalo irresistible, pero una vez que estamos literalmente enganchados, damos a cambio un bien precioso: toda la información que las compañías necesitan para ajustar a nuestro gusto los bienes de consumo y los servicios que quieren vender. Hay otras cuestiones en juego. No se trata sólo de la apropiación del trabajo gratuito y de la obvia pérdida de privacidad. De hecho, puede pensarse que la noción burguesa de privacidad está condenada a cambiar de manera radical o a desaparecer con las nuevas dinámicas creadas por el capitalismo electrónicoinformático. Además de la capacidad que estas compañías tienen de esconder la fuente de sus ingresos, hay una cuestión política en juego. Me refiero a las relaciones íntimas que median entre estas empresas de recolección de información, la elite política y el aparato de seguridad de Estados Unidos. ${ }^{4}$ Asimismo, el orden de los resultados de búsqueda puede tener consecuencias políticas y se ha mostrado que los algoritmos de Google tienen un sesgo conservador de derecha y que las búsquedas pueden ser manipuladas con frecuencia por derechistas expertos en tecnología (Cadwalladr, 2016).

Google también ha sido considerado el "principal agente de la red de información" (Gerlitz y Helmond, 2011: 2), un término "usado para describir la world wide web como un medio [...] para publicar contenido, caracterizado por el enlace de información" (2011: 1). Gerlitz y Helmond ven el enlace — link - como una moneda interna en la "red de informaciones", en la que los contadores son herramientas importantes para la atribución de valor. Sergey Brin y Larry Page, fundadores de Google, crearon el PageRank, un algoritmo de análisis de hiperenlaces que clasifica el valor de una página web de acuerdo con los números de enlaces

En 2012, Google obtuvo 96\% de sus ingresos de los anuncios (McFarlane, 2012).

3 En 2008, apenas en Estados Unidos, se hicieron 4.4 mil millones de búsquedas por mes, 6112000 veces por hora (Jarvis, 2011: 28). En 2016, estos números eran al menos 63000 búsquedas por segundo, 5.5 mil millones por día (Cadwalladr, 2016). El papel del trabajo no pagado en la "economía digital" fue explorado por Terranova (2000).

$4 \quad$ "Uno de los fundadores de Facebook, Chris Hughes, fue un asesor de la campaña [de 2008] de Obama” (Jarvis, 2011: 51). 
que recibe. Una vez más, el capitalismo electrónicoinformático crea su propia mercancía:

El algoritmo PageRank ha establecido una economía gobernada por motores de búsqueda que regulan el valor de cada enlace (Walker, 2002). Después llevó a una mercantilización de los enlaces como objetos de la red que pueden negociarse, venderse o comprarse al interior de la economía de los enlaces (Gerlitz y Helmond, 2011: 4).

La práctica de conectar información y contenido para clasificarlos con la ayuda de motores de búsqueda comenzó a tener una fuerte competencia con el surgimiento de las "redes sociales" — social web - o lo que Gerlitz y Helmond llaman la "economía de los clics" - hit economy —, que impulsa la "economía del me gusta" — like economy-. Aquí los "motores de búsqueda y las plataformas de los social media observan las conexiones entre las personas y sus relaciones con otros usuarios de la red u objetos de la red" (2011: 3). Si Google es el "regulador de la economía de los enlaces" de la red de información, Facebook, por su parte, es el principal actor de la "red social" en la que crece la "economía del me gusta" (2011: 2). Los botones de "me gusta" y "compartir", también reciben el nombre de botones sociales: permiten contar las reacciones ante ciertos contenidos, y sobre todo, vincular estas preferencias con redes e individuos específicos. De una manera congruente con la tendencia del capitalismo electrónico-informático a apropiarse de manera gratuita del trabajo, los usuarios y las conexiones que éstos crean son los principales bienes de Facebook (2011: 18):

Mientras los enlaces de la red de información fueron creados por webmasters y clasificados de acuerdo con el PageRank de Google, en la red social, los enlaces son creados por los usuarios, que, al mismo tiempo, les agregan valor por medio del me gusta y comentarios [...]. Lo que está en juego aquí no es sólo quién crea los enlaces en la red, sino también cómo y quiénes califican estos enlaces [...], la economía del me gusta cambia la visibilidad de los enlaces (2011: 13).

Los plugins y botones de Facebook funcionan como cookies que permiten rastrear el comportamiento de los usuarios en la red. Sin saberlo, los usuarios, además de brindar información sobre sus propios perfiles, contribuyen a la "re-centralización del tejido de la red y de los flujos de información y asociación afectiva" (Gerlitz y Helmond, 2011: 19). Facebook y otras plataformas de social media "permiten [...] actividades contabilizables como el tuitear, el me gusta, el compartir o el comentar" que convierten las "respuestas afectivas" y "dinámicas sociales" en valores comparables (2011: 21). Gerlitz y Helmond concluyen lo siguiente:

Sobre todo en contextos de marketing, las actividades de los social media son cuidadosamente rastreadas, monitoreadas y analizadas, por algoritmos o por investigadores humanos con la ayuda de herramientas. Así, extrapolando la idea de que las actividades de las redes sociales funcionan como una moneda en la economía del me gusta, podemos decir que se trata de una moneda de altos números, social e informacional a un tiempo. Además, esta moneda es también altamente ubicua y técnica, ya que el Open Social Graph crea una infraestructura donde todo comportamiento en la red contribuye a las prácticas de minería de datos de Facebook. Sin importar si un usuario de la red decide participar en Facebook o no, la tecnicidad de los botones sociales convierte a los usuarios de la red en partícipes de la economía del me gusta, que producen constantemente valiosos datos de usuario y contribuyen a la indexación social sin siquiera saberlo (2011: 25).

En el interior de la economía de la carnada, la publicidad y la recomendación personalizada que 
emergen de una red son las fuerzas que operan tras la seducción de los consumidores. El objetivo a largo plazo es prever los intereses de los consumidores. En resumen, una vez que uno está enganchado en una plataforma de redes sociales, cada uso de un botón social, comentario, foto o video publicado es trabajo gratis para estas corporaciones y significa que uno les está ayudando a convertirse en monopolios del capitalismo electrónico-informático. En la economía digital, el motor principal es el trabajo no remunerado de los usuarios. En este universo, el huésped ignora al parásito o no se preocupa por él.

Pero, ¿cómo se llevan a cabo estas operaciones de seleccionar y organizar información para usarla como "anuncios y recomendaciones dirigidos personalmente"? El adverbio "personalmente" debería incluir las redes de la persona, porque nadie es sólo una persona en la red social. Aquí vuelve a surgir la dependencia del capitalismo electrónico-informático del conocimiento científico y técnico. Algunas veces esta dependencia parece contradecir las historias de vida de figuras míticas y fundadoras, como Zuckerberg o Jobs, que abandonaron sus estudios universitarios para contarse entre los hombres más ricos del mundo. Se dice, por ejemplo, que Zuckerberg es un geek y se supone que los geeks van a "tomar el control del mundo". Cuando esto ocurra, "unas pocas palabras contundentes y una mirada silenciosa se convertirán en una norma social", afirma Jeff Jarvis (2011: 48), un ideólogo del capitalismo electrónico-informático. En este medio, también hay una tendencia a creer que cuanto más joven sea la persona, más capaz será de innovar. De cualquier manera, es imposible olvidar el papel que ha jugado y sigue jugando la Universidad de Stanford en el desarrollo general de Silicon Valley. De hecho, la minería de datos depende mucho de los algoritmos y aquí es donde los matemáticos se suman a los ingenieros y la ciencia se fusiona una vez más con la dinámica industrial. ${ }^{5}$ Nicholas Diakopoulos describe así el poder de los algoritmos en la vida actual:
Vivimos en un mundo donde los algoritmos, y los datos que los alimentan, son los árbitros en una gran cantidad de decisiones de nuestras vidas: no sólo los motores de búsqueda y los sistemas personalizados de noticias online, sino también las evaluaciones educativas, el funcionamiento de los mercados y de las campañas políticas, el diseño de espacios públicos urbanos, e incluso la forma en que se administran servicios sociales como la seguridad social y la seguridad pública. Pero los algoritmos pueden cometer errores y operar con sesgos. La opacidad de los algoritmos técnicamente complejos que operan a escala dificulta su escrutinio, y ello produce una falta de claridad para el público en lo que respecta a la forma en que ejercen su poder e influencia (2014: 398).

Hoy en día, los algoritmos son herramienta importante de lo que Foucault llamó biopolítica: representan "una nueva tecnología de poder [que] existe en un nivel diferente, en una escala diferente, y tiene un área de incidencia diferente y hace uso de instrumentos muy diferentes" (2003: 242). Los algoritmos "necesitan la datificación del mundo", "problematizan la vida pública" y con frecuencia tienen "capacidad de decisión autónoma”, y ello comprende la priorización, clasificación, asociación y filtración (Diakopoulos, 2014: 400). Todas estas actividades implican valores, sesgos e intenciones de los agentes y agencias que diseñan los algoritmos. La transparencia y la rendición de cuentas, por lo tanto, se convierten en las principales necesidades para controlar los excesos de los algoritmos. No es fácil implementar estas medidas, porque las compañías dependen del secreto para mantener su competitividad. Google, por ejemplo, a pesar de trabajar con una enorme cantidad de información en internet, es

"Un algoritmo puede definirse como una serie de pasos que se dan para resolver un problema particular o alcanzar un resultado definido" (Diakopoulos, 2014: 400). 
una compañía con un alto grado de secrecía (Levy, 2011: 354). Los algoritmos, por lo tanto, son una característica central de la hegemonía del capitalismo electrónico-informático. Concuerdo con Scott Lash cuando reconoce el poder generalizado de las reglas algorítmicas y las ve como "caminos a través de los cuales funciona el poder capitalista". Lash concluye que "una sociedad en la que los medios son omnipresentes es una sociedad en la que el poder reside, cada vez más, en el algoritmo" (2007: 71).

\section{Googleísmo}

Con frecuencia se piensa en la historia del capitalismo industrial como una secuencia de modos de organizar y administrar los factores de producción para alcanzar una mayor racionalidad en los procesos de producción e incrementar los beneficios obtenidos. El taylorismo fue un paso importante en esta evolución del proceso laboral y la cultura gerencial, lo mismo que el fordismo y el toyotismo. Hay que notar que estos rótulos se refieren a la persona o compañía que inventó el modelo ideal de estas técnicas gerenciales. La mayor parte de las veces estas innovaciones gerenciales implican la apropiación de conocimientos técnicos preexistentes por parte de elites supuestamente clarividentes que las presentan como una revolución económica, política y social. Se convierten, así, en modelos, es decir, en una "codificación de paradigmas que salen a la luz en países industrializados avanzados" (Bueno, 2016: 34), centros a partir de los cuales se difunden en el ámbito internacional. Para mí, el googleísmo es la nueva moda del mundo empresarial.

Los ideólogos del googleísmo abundan. ${ }^{6}$ De vez en cuando, ni siquiera los que estudian la era digital pueden dejar de hacer declaraciones hiperbólicas sobre Google. Martina Mahnke y Emma Uprichard, por ejemplo, dicen:
A veces es difícil imaginar qué sería de la vida sin Google. ¿Qué haríamos? ¿Utilizar otro motor de búsqueda? ¿Dejar de interesarnos por la información en internet? Tratar de responder a estas preguntas, por hipotéticas que sean, nos lleva a la conclusión de que Google es más que una herramienta, es un mito moderno (2014: 258).

Los ideólogos crean o consolidan nuevas visiones y palabras de moda. "Google es nuestro modelo para pensar de nuevas maneras porque es singularmente exitoso", escribie Jeff Jarvis (2011: 5) en su éxito de ventas ¿Qué haría Google? Jarvis es un empresario de internet, un profesor de periodismo en la Universidad de la Ciudad de Nueva York, en la que preside el Centro para el Periodismo Empresarial, y es un autor al que el Foro Económico Mundial de Davos considera entre los 100 líderes mundiales de los medios de comunicación. Entre las reglas de la "nueva era", Jarvis (2011: 4-5) incluye la muerte del mercado de masas, ahora reemplazado por la masa de los nichos; la sustitución del marketing por la conversación, como la nueva habilidad de las organizaciones, y lo que es más interesante, afirma que permitir que los "clientes colaboren con usted - en la creación, distribución, marketing y soporte de los productos - es lo que crea una ventaja en el mercado actual" (2011: 3-4). Se supone que estamos siendo testigos del fin de los sistemas de control y que el término apertura se ha convertido en una palabra clave. La negación del libre acceso a los servicios significa que se está desperdiciando la enorme creatividad que hay en la multitud virtual.

6

Hay incluso una Iglesia de Google. Ellos piensan que "Google es lo más cerca que la humanidad ha estado de experimentar directamente a un Dios real (como se define de manera típica). Creemos que hay mucha más evidencia a favor de la divinidad de Google de la que hay para la divinidad de otros dioses más tradicionales". Véase <http:// churchofgoogle.org>. Consultado el 8 de febrero de 2017. 
Y la creatividad es la fuente de la innovación. Jarvis enfatiza que los usuarios siempre están haciendo a Google más inteligente y que la compañía "explora la sabiduría de la multitud” y organiza la inmensa cantidad de conocimiento disponible en la red (2011: 6). El libro de Jarvis está lleno de aforismos que pretenden describir la nueva economía: "tu peor cliente es tu mejor amigo"; "tu mejor cliente es tu socio"; "el enlace lo cambia todo"; "haz lo que mejor sabes hacer y enlaza el resto"; "si no eres buscable, nadie te encontrará"; "Google piensa distribuido. Viene a nosotros cuando y como puede" (2011: 36) y empodera al usuario como sujeto de la red. No nos sorprende que se alabe al networking y la descentralización como modalidades de organización empresarial junto con las "plataformas" en una nueva ronda de glorificación del mercado libre y la competencia.

Al igual que en otros momentos, en los que un determinado sector industrial estableció su hegemonía, se postulan nuevas verdades y panaceas como formas inevitables de salir adelante en el "nuevo orden mundial", en una "economía posescasez" (2011: 57). Jarvis lo explica de este modo:

Ya no necesitamos a las empresas, las instituciones o los gobiernos para que nos organicen. Ahora tenemos herramientas para organizarnos nosotros mismos. Podemos encontrarnos y unirnos en torno a causas políticas, malas compañías, talentos, negocios o ideas. Podemos compartir y categorizar nuestro conocimiento y comportamiento [...]. También tenemos nuevas éticas y actitudes que surgen de esta nueva organización y cambian a la sociedad de maneras que todavía no podemos comprender, con apertura, generosidad, colaboración y eficiencia. Estamos usando el tejido conectivo de internet para saltar por encima de las fronteras — ya sea que rodeen países, compañías o estadísticas demográficas- (2011: 52-53).

Jarvis llama "economía del código abierto, del don" a lo que yo llamo economía de la carnada. Su definición de "economía del don" haría que Marcel Mauss se revolcara en su tumba. ${ }^{7}$ La explicación de la existencia de Linux, el software libre más importante, es la "generosidad" y no que se basa en un movimiento político que ha luchado contra el software protegido por patentes. Los programadores trabajan gratis porque "les importa" y "quieren ser parte de algo". Los que alimentan Wikipedia lo hacen por "generosidad y ego". Parece que el capitalismo electrónico-informático ha encontrado un rico filón. Las ideas, la fuente de la invención, la innovación y la diferenciación son gratis en internet. Es más, hoy en día, los capitalistas pueden tener acceso al megacerebro global que genera creatividad a una escala y una velocidad sin precedentes. La palabra de moda para referirse a este movimiento es crowdsourcing.

Crowdsourcing quiere decir, de manera literal, "externalizar para la multitud: un modelo de producción que usa la inteligencia y el conocimiento voluntario y colectivo difundido por internet para resolver problemas, crear contenido o desarrollar nuevas tecnologías" (Moulier-Boutang, 2010: 75). Pero también consiste en "hacer tareas simples, repetitivas, como etiquetar imágenes, transcribir materiales de audio o seleccionar registros de bases de datos en línea" (Howe, 2009: XIV). El crowdsourcing también puede verse como "un cottage-system electrónico y global”, es decir, como una amplificación de los movimientos de descentralización que el capital ha llevado a cabo desde la primera Revolución industrial, cuando las fábricas de textiles explotaban a las personas y las ponían a trabajar en sus casas.

7

"La economía del don es más que sólo escuchar a los clientes por cortesía o respeto. Se trata de comprender que los clientes [...] quieren tener una voz y ganar control. Es una mejor manera de hacer negocios. ¿Pueden los clientes ayudar a diseñar los productos? ¿Pueden los ciudadanos ayudar a escribir leyes? ¿Pueden asignar periodistas?" (Jarvis, 2011: 62). 


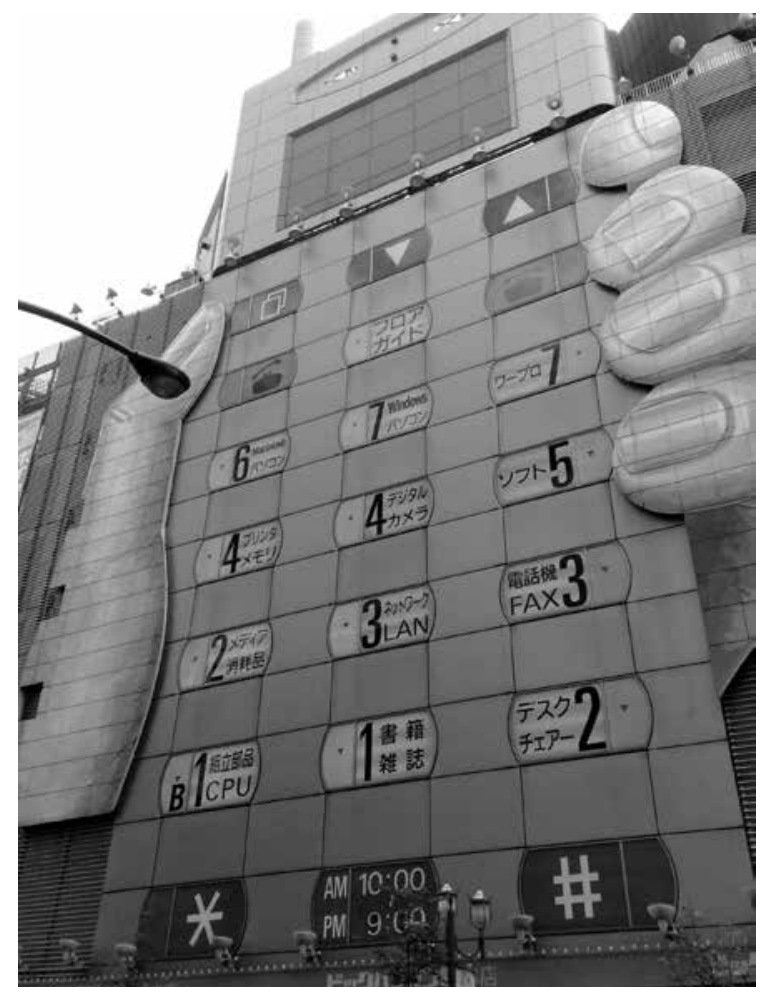

JAMES NASH - Tienda de teléfonos celulares en Ikebukuro, Tokio, Japón, octubre de 2008.

La diferencia ahora no se limita al alcance del cottagesystem - éste se ha vuelto global y funciona las 24 horas, los siete días de la semana-, sino al hecho de que millones de personas representan una fuerza de trabajo remunerada, o con frecuencia no remunerada, que trabaja para mejorar la competitividad de las empresas capitalistas. El mundo virtual ha resemantizado el significado de la multitud y sus capacidades. Para Jeff Howe, "si se da un conjunto adecuado de condiciones, la multitud casi siempre superará cualquier número de empleados —un hecho que las empresas empiezan a tener presente y que tratan de explotar cada vez más_-” (2009: 11). La multitud virtual se ve como productora de "una especie de meritocracia perfecta. Ya no importan el pedigrí, la raza, el género, la edad o la calificación. Lo que queda es la calidad del trabajo mismo" (2009: 13).
Una de las virtudes de la "innovación en masa" en línea es que "facilita el trabajo móvil y la comunicación multisituada, superando los obstáculos para la colaboración a distancia y el acoplamiento de procesos que operan de manera simultánea" (Bueno, 2016: 225). Las nuevas habilidades cognitivas, capacidades multitareas y la posibilidad de cooperación interdisciplinaria caracterizan el ambiente colaborativo que produce nuevos sentidos de comunidad en los que se rompen los límites institucionales y organizacionales (2016: 226-228). Los clientes se convierten en una fuente de innovación y perfeccionamiento, algo sintetizado por la expresión “contenido generado por los usuarios". Una gran cantidad de científicos, profesionales o aficionados dispersos por todo el mundo pueden encontrar soluciones para el desarrollo de nuevos productos y servicios que los empleados de la compañía no han podido encontrar (Howe, 2009).

En realidad, el crowdsourcing involucra: a) las personas que cooperan de forma gratuita, inspiradas en nociones abstractas de comunidad o en valores políticos - el ejemplo clásico y origen del crowdsourcing es el Movimiento Software Libre-; b) las personas que están empleadas y utilizan su tiempo libre para resolver problemas que se publican en internet y obtener algo de dinero extra; c) las personas que se dedican por completo a este tipo de actividad para ganarse la vida. Aquí sale a la luz un nuevo tipo de trabajador, el trabajador sin cuello - no collar labour - (Ross, 2001, citado en Bueno, 2016: 244). Se trata de personas que visten de manera informal, pueden trabajar en sus dormitorios y reunirse en cafeterías, y tienen "un nuevo ethos que confunde la vida con el trabajo, generando ambientes geniales, casi lúdicos". Estos trabajadores se explotan a sí mismos y a sus familias (Bueno, 2016: 244), pero encomian la colectividad, la creatividad, la cooperación, la participación, el intercambio y el trabajo en red. Sin embargo, la ausencia de derechos laborales se dramatiza en términos como "precariado" 
y "esclavos de la red" (Terranova, 2000; Bueno, 2016: 245), utilizados para describir a los miembros de la llamada clase creativa. Al mismo tiempo, el fantasma de los despidos y el desempleo persigue al comercio tradicional y las ocupaciones laborales tradicionales, ya que las compañías de crowdsourcing se especializan en ofrecer sus servicios a todo tipo de empresas, y el cottage-system electrónico global aumenta la competencia generalizada.

De hecho, Google no sólo inspira modelos de negocios y de producción de ganancias, sino también un nuevo modelo de relaciones laborales y cultura administrativa. El consultor administrativo Bernard Girard escribió un libro en el que compara el papel de Google con los que desempeñaron Ford y Toyota en la administración industrial, con el fin de mostrar que esta compañía es "un modelo de gestión para la economía del conocimiento” (2009: 3): ${ }^{8}$

Google puede verse como un nuevo arquetipo de empresa, porque su gestión ha hecho varias innovaciones en términos de recursos humanos, producción, relación con los clientes y, sobre todo, en lo que concierne al control de sus operaciones de producción [...]. El rápido crecimiento de la compañía, la personalidad de sus fundadores, su visión, su cultura científica, sus obsesiones y el expertise que la rodea han fomentado la construcción de este modelo único de negocios que es el estilo Google (2009: 2).

Girard (2009: 223) insiste y afirma que "no es una exageración" llamar revolucionario al nuevo modelo gerencial de Google:

Esta revolución también tiene una dimensión social. Rara vez alguna empresa ha confiado tanto como Google en el "capital voluntario" de sus trabajadores, sus contactos y relaciones para probar nuevos productos o para obtener nuevas ideas y mejorar productos. Sin duda, Google es la primera compañía que descubrió la forma de beneficiarse del desarrollo de comunidades de fans compuestas no sólo por amantes de la causa pero también por observadores y críticos sin piedad (que son las fuentes de información más efectivas de Google, precisamente porque sus críticas son muy severas) (2009: 224).

Google es una empresa global que está presente en más de 70 países. Su sede mundial, ubicada en Mountain View, en Silicon Valley, California, se conoce como el Googleplex. Es un lugar cosmopolita, una Meca para los jóvenes talentosos, entusiastas de las oportunidades abiertas por el capitalismo electrónico-informático. Google tiene sus sesgos, por ejemplo, reclutar personas educadas en universidades de elite, con doctorados y altos puntajes en las pruebas educativas (Poundstone, 2012: 56). Según William Poundstone, autor del libro ¿Eres lo suficientemente inteligente para trabajar en Google?, el Plex es "donde la gente más inteligente hace las cosas más padres" (2012: 5). La revista Fortune (citada en Poundstone, 2012) pone a Google "en la cima o cerca de la cima”, entre las "100 mejores compañías para trabajar" (2012: 5). En 2012, Google recibió un millón de solicitudes de empleo, pero sólo uno de cada 130 solicitantes consiguió el empleo (2012: 4). A pesar del valor concedido a la descentralización, la aprobación final de los nuevos empleados siempre se somete al Googleplex, ubicado en California (2012: 55), sin importar de qué país provienen los trabajadores o cuál será su lugar de trabajo.

El ambiente de trabajo es famoso porque parece un club con una "enorme cantidad de comodidades para sus empleados, presumiblemente afortunados" (2012: 5).

8

Para una discusión sobre la transición del fordismo al toyotismo y la diseminación internacional de este último, véase Bueno (2016: 137-162). 
Hay 11 restaurantes gourmet que sirven comida gratis, orgánica, cultivada localmente, paredes de escalar, albercas, pizarras gigantes para compartir pensamientos espontáneos, ping-pong, futbolito [...], cabinas telefónicas británicas, plantas podadas en forma de dinosaurios. Los empleados de Google tienen acceso gratuito a lavadoras, a vacunas antigripales, clases de idiomas, lavado de coches y cambios de aceite. Hay servicio de transporte entre el hogar y el trabajo, un descuento de 5000 dólares en la compra de coches híbridos, scooters comunitarios para el uso de cualquier persona en el campus. Los nuevos padres y madres reciben 500 dólares en comida para llevar y 18 semanas de permiso para crear vínculos con su bebé. Google paga el impuesto sobre la renta para parejas del mismo sexo. Todos los empleados reciben un viaje anual para esquiar. Estas ventajas no se deben necesariamente a la generosidad y, a diferencia de las conquistas que generaciones anteriores alcanzaron en sus lugares de trabajo, no fueron negociadas por sindicatos o individuos. Es un buen negocio para Google ofrecer estos beneficios en una industria que depende tanto de la atracción de los mejores talentos. Estos beneficios no sólo mantienen a los empleados felices, sino que también mantienen a todos los demás con la nariz presionada contra el cristal (2012: 5-6).

El sistema de reclutamiento de Google se basa en entrevistas. Los solicitantes se someten a procesos de selección cada vez más invasivos y a entrevistadores que actúan como oráculos, que hacen preguntas absurdas con las que, según ellos, identifican a los genios innovadores con mentes flexibles e increíbles, capaces de encontrar soluciones visionarias para diferentes tipos de problemas, y con habilidades empresariales. Las palabras clave son, aquí, flexibilidad mental, creatividad y capacidad para innovar. El capitalismo electrónico-informático hiperflexible necesita una mano de obra flexible y creativa. Google, una compañía que en última instancia aspira a centralizar todo el conocimiento e información del mundo, no sólo busca creatividad e innovación gratuitas disponibles en la red, sino que establece un esquema de reclutamiento y un ambiente de trabajo supuestamente capaces de extraer más creatividad y producir más innovación. En sintonía con el reconocimiento de la creatividad como una fuerza productiva, los ingenieros pueden trabajar un día a la semana en un proyecto que ellos elijan, una iniciativa que es responsable de las ideas que están detrás de más de la mitad de los ingresos de la compañía (2012: 12).

En resumen, por un lado, Google se apropia del conocimiento y la creatividad en internet de manera gratuita. Por el otro, pretende crear una mística laboral y un ambiente de trabajo en el que parece que los empleados no están trabajando, que no están en un lugar de trabajo, sino ejerciendo su creatividad e imaginación en un club.

\section{Comentarios finales}

En 2014, tres de las cinco empresas con los mayores valores bursátiles representaban la actual hegemonía del capitalismo electrónico-informático (Wright y Pasquali, 2015). Apple estaba en primer lugar, y Microsoft y Google en el tercer y cuarto lugar, respectivamente. Google saltó 11 posiciones de 2013 a 2014. Apple y Microsoft son muy dependientes de los derechos de la propiedad intelectual, al tiempo que Google es menos dependiente, y eso hace de Google y de Facebook la vanguardia del capitalismo electrónico-informático. Mi elección de Google como la principal empresa a analizar refleja su papel de liderazgo, así como el alcance y la naturaleza de sus operaciones. De hecho, el poder de Google se basa en gran medida en la capacidad de aprovechar grandes cantidades de creatividad y trabajo gratis tanto en el mundo virtual como en el real. Dado que la principal mercancía de ésta y otras empresas 
líderes del capitalismo electrónico-informático es la información, el libre flujo de información es un factor clave para establecer y consolidar su hegemonía. Por un lado, esto significa que los copyrights y patentes, aunque todavía son importantes, se conciben de una manera más flexible según los intereses más inmediatos de las compañías. Es bien sabido, por ejemplo, que Google luchó por liberar los derechos de autor cuando le interesaba copiar libros para crear la biblioteca virtual más grande y completa del mundo. Por otro lado, cuando un interés importante para Google necesita ser resguardado por patentes y secretos, la compañía así lo hará.

Al mismo tiempo, las empresas líderes del capitalismo electrónico-informático han establecido o consolidado nuevos modelos gerenciales que se exportan a otras actividades económicas y se difunden como panaceas de la vida económica, social, política y cultural. El éxito de las empresas líderes mencionadas en este artículo se basa también en el descubrimiento de nuevos recursos económicos — como la mercantilización de la palabra por Google- y en la apropiación, global, del conocimiento y el trabajo gratuitos de sus usuarios, de las acciones de sus usuarios transformadas en fuentes gratuitas de valor, lo que presento aquí como la economía de la carnada, que se subdivide a su vez en la economía de los enlaces, los clics y el me gusta. Su éxito también depende de su impresionante crecimiento en los mercados financieros y en el establecimiento de nuevas relaciones laborales. Al igual que en otros momentos de la historia del capitalismo industrial, las figuras míticas de esta rama de actividades se ven como pioneros que definen las tendencias del futuro. Todo indica que la hegemonía del capitalismo electrónico-informático seguirá en aumento. D

\section{Bibliografía}

Albagli, Sarita y Maria Lúcia Maciel, 2010, “Information, Power, and Politics: From the South, Beyond the South”, en Sarita Albagli y Maria Lúcia Maciel (eds.), Information, Power and Politics. Technological and Institutional Mediations, Lexington Books, Plymouth, pp. 1-25.

Anderson, Benedict, 1983, Imagined Communities. Reflections on the Origin and Spread of Nationalism, Verso, Londres.

Benner, Chris, 2002, Work in the New Economy: Flexible Labor Markets in Silicon Valley, Blackwell Publishers, Malden.

Blondeau, Olivier et al., 2004, Capitalismo cognitivo, propiedad intelectual y creación colectiva, Traficantes de Sueños, Madrid.

Bourdieu, Pierre, 1986, Questions de sociologie, Éditions de Minuit, París.

Braverman, Harry, 1974, Labor and Monopoly Capital, Monthly Review Press, Nueva York.

Bueno Castellanos, Carmen, 2016, Configuraciones productivas en la globalización: trayectorias a la mexicana, Centro de Investigaciones y Estudios Superiores en Antropología Social/Universidad Iberoamericana, México.

Cadwalladr, Carole, 2016, "Google, Democracy and the Truth about Internet Search", en The Guardian, 4 de diciembre. Disponible en línea: <https://www.theguardian.com/technology/2016/dec/04/google-democracy-truth-internet-search-facebook?CMP=share_btn_fb>. Consultado el 21 de diciembre de 2016.

Castells, Manuel, 1989, The Informational City. Information Technology, Economic Restructuring and the Urban Regional Process, Blackwell, Oxford.

, 1996, The Rise of the Network Society, Blackwell, Oxford.

Cocco, Giuseppe, Gerardo Silva y Alexander Patez Galvão, 2003, Capitalismo cognitivo: trabalho, redes e inovação, DP\&A, Río de Janeiro. Corsani, Antonella, 2003, “Elementos de uma ruptura: a hipótese do capitalismo cognitivo”, en Giuseppe Cocco, Gerardo Silva y Alexander Patez Galvão (eds.), Capitalismo cognitivo: trabalho, redes e inovação, DP\&A, Río de Janeiro, pp. 15-32.

Diakopoulos, Nicholas, 2014, "Algorithmic Accountability", en Digital Journalism, vol. 3, núm. 3, pp. 398-415.

Engels, Friedrich, 2009 [1844], The Condition of the Working Class in England, Oxford University Press, Oxford.

Foucault, Michel, 2003, Society Must Be Defended. Lectures at the Collège de France, 1975-76, Picador, Nueva York.

Fumagalli, Andrea, 2010, Bioeconomía y capitalismo cognitivo. Hacia un nuevo paradigma de acumulación, Traficantes de Sueños, Madrid. 
Gerlitz, Carolin y Anne Helmond, 2011, "Hit, Link, Like and Share. Organizing the Social and the Fabric of the Web in a Like Economy", ponencia presentada en la miniconferencia de Digital Methods Initiative, Universidad de Ámsterdam, 24 y 25 de enero.

Girard, Bernard, 2009, The Google Way. How One Company Is Revolutionizing Management as We Know It, No Starch Press, San Francisco. Gramsci, Antonio, 2006, Cadernos do cárcere, Civilização Brasileira, Río de Janeiro.

Hesmondhalgh, David, 2016, "Exploitation and Media Labor", en Richard Maxwell (ed.), The Routledge Companion to Labor and Media, Routledge, Nueva York, pp. 30-39.

Howe, Jeff, 2009, Crowdsourcing. Why the Power of the Crowd Is Driving the Future of Business, Three Rivers Press, Nueva York. Jarvis, Jeff, 2011, What Would Google Do? Reverse-Engineering the Fastest-Growing Company in the History of the World, Harper, Nueva York. Lash, Scott, 2007, "Power after Hegemony: Cultural Studies in Mutation?", en Theory Culture \& Society, vol. 24, núm. 3, pp. 55-78.

Latham, Robert y Saskia Sassen, 2005, "Digital Formations. Constructing an Object of Study”, en Robert Latham y Saskia Sassen (eds.), Digital Formations. IT and New Architectures in the Global Realm, Princeton University Press, Princeton, pp. 1-33.

Levy, Steven, 2011, In the Plex. How Google Thinks, Works and Shapes Our Lives, Simon \& Schuster, Nueva York.

Lins Ribeiro, Gustavo, 1998, "Cybercultural Politics. Political Activism at a Distance in a Transnational World", en Sonia Alvarez, Evelina Dagnino y Arturo Escobar (eds.), Cultures of Politics/Politics of Cultures. Revisioning Latin American Social Movements, Westview Press, Boulder, pp. 325-352.

2003, Postimperialismo. Cultura y política en el mundo contemporáneo, Gedisa, Barcelona.

Lunden, Ingrid, 2015, “6.1B Smartphone Users Globally by 2020, Overtaking Basic Fixed Phone Subscriptions”, en Tech Crunch, 2 de junio. Disponible en línea: < https://techcrunch.com/2015/06/02/6-1b-smartphone-users-globally-by-2020-overtaking-basic-fixed-phonesubscriptions/>. Consultado el 10 de marzo de 2016.

Macciocchi, Maria Antonietta, 1974, “Hégémonie, bloc historique, État”, en Maria Antonietta Macciocchi, Pour Gramsci, Éditions du Seuil, París, pp. 158-202.

Mahnke, Martina y Emma Uprichard, 2014, "Algorithming the Algorithm”, en René König (ed.), Society of the Query Reader. Reflections on Web Search, Institute of Network Cultures, Ámsterdam, pp. 256-270.

McFarlane, Greg, 2012, “How Does Google Make Its Money?”, en Investopedia, 2 de noviembre. Disponible en línea: <http://www.investopedia. com/stock-analysis/2012/what-does-google-actually-make-money-from-goog1121.aspx>. Consultado el 21 de diciembre de 2016.

Moulier-Boutang, Yann, 2008, Le capitalisme cognitif, la nouvelle grande transformation, Éditions Amsterdam, París.

, 2010, "Wikipolitics and the Economy of the Bees: Information, Power, and Politics in a Digital Society", en Sarita Albagli y Maria Lucia Maciel (eds.), Information, Power and Politics. Technological and Institutional Mediations, Lexington Books, Plymouth, pp. 47-77.

Mumford, Lewis, 1950, Technique et civilisation, Éditions du Seuil, París.

Noble, David F., 1977, America by Design. Science, Technology, and The Rise of Corporate Capitalism, Oxford University Press, Oxford.

Poundstone, William, 2012, Are you Smart Enough to Work at Google?, Little, Brown \& Company, Nueva York.

Rogers, Richard, 2002, "Operating Issue Networks on the Web”, en Science as Culture, vol. 11, núm. 2, pp. 191-213.

Ross, Andrew, 2001, “No Collar Labour, in America’s 'New Economy”, en Socialist Register, núm. 37, pp. 77-87.

Saxenian, AnnaLee, 2016, "El modelo Silicon Valley: dinamismo económico, exclusión social”, en Manuel Castells y Pekka Himanen (eds.), Reconceptualización del desarrollo en la era global de la información, Fondo de Cultura Económica, México, pp. 47-69.

Schiller, Dan, 2000, Digital Capitalism. Networking the Global Market System, MIT Press, Cambridge.

__ 2016, "Labor and Digital Capitalism”, en Richard Maxwell (ed.), The Routledge Companion to Labor and Media, Routledge, Nueva York, pp. 3-17.

Schmidt, Alfred, 1977, El concepto de naturaleza en Marx, Siglo XXI Editores, Madrid.

Terranova, Tiziana, 2000, "Free Labor: Producing Culture for the Digital Economy", en Social Text, vol. 18, núm. 2, pp. 33-58.

Thomson, Ross, 2009, Structures of Change in the Mechanical Age: Technological Innovation in the United States, 1790-1865, The Johns Hopkins University Press, Baltimore.

Vercellone, Carlo, 2011, Capitalismo cognitivo, renta, saber y valor en la época posfordista, Prometeo, Buenos Aires.

Walker, Jill, 2002, "Links and Power: The Political Economy of Linking on the Web”, en Kenneth M. Anderson, Stuart Moulthrop y James Blustein (eds.), Hypertext 2002. Proceedings of the Thirteenth ACM Conference on Hypertext and Hypermedia, Acm Press, Nueva York, pp. 72-73.

Williams, Raymond, 1983, Keywords. A Vocabulary of Culture and Society, Oxford University Press, Nueva York.

Wright, Gilly y Valentina Pasquali, 2015, “World’s Largest Companies”, en Global Finance, 19 de noviembre. Disponible en línea: <http://www. gfmag.com/global-data/economic-data/largest-companies?page=4>. Consultado el 8 de abril de 2016 . 